\title{
Transformer la recherche en santé au Canada en tenant compte de la race
}

\author{
Geetanjali Datta MSP DSc, Arjumand Siddiqi MSP DSc, Aisha Lofters MD PhD
}

Citation : CMAJ 2021 January 18;193:E99-100. doi : 10.1503/cmaj.201742-f

Voir la version anglaise de l'article ici : www.cmaj.ca/lookup/doi/10.1503/cmaj.201742

$\mathbf{L}$ a pandémie de maladie à coronavirus 2019 a fait éclater au grand jour des lacunes dans nos structures sociales, sources d'inégalités en santé. Elle a aussi révélé les failles des infrastructures du Canada sur le plan de la détection et de la réduction des inégalités raciales dans les résultats de santé. Le Canada a tardé à reconnaître le rôle du racisme structurel et sa contribution à la création et à la perpétuation d'inégalités dans la société, y compris en santé. Or pour renforcer nos capacités de recherche sur la mesure et l'élimination des inégalités raciales dans ce secteur, des mesures décisives s'imposent. En tant que principal organisme de financement de la recherche en santé au pays, les Instituts de recherche en santé du Canada (IRSC) ont le pouvoir et le devoir d'agir en ce sens, en créant un Institut du racisme et de ses effets sur la santé.

Les racines historiques de ce mal structurel au Canada sont ancrées dans son passé colonial, époque où les Autochtones et les personnes d'ascendance africaine ont été réduits à l'esclavage ${ }^{1,2}$. Nous en subissons encore les répercussions, comme en témoigne le Groupe de travail d'experts sur les personnes d'ascendance africaine dans son rapport sur sa mission au Canada en $2017^{3}$. Son constat? Malgré l'excellente réputation du Canada en matière d'inclusion, "le racisme envers les Noirs et les stéréotypes raciaux [...] sont tellement enracinés profondément dans les institutions, les politiques et les pratiques que leurs manifestations institutionnelles ou systémiques sont soit fonctionnellement normalisées, soit occultées, surtout du point de vue du groupe dominant ${ }^{3}$. » En 2018, l'Association canadienne de santé publique a reconnu avoir contribué à perpétuer du racisme systémique et a indiqué dans son rapport que le racisme envers les Noirs, " omniprésent dans de nombreuses institutions canadiennes, est responsable de la perpétuation contemporaine de la pauvreté et du chômage, du profilage racial, de la violence policière, des incarcérations, de la détention liée à l'immigration, des expulsions, des pratiques d'exploitation des travailleurs migrants, des placements disproportionnés d'enfants et des faibles taux de diplomation ${ }^{4}$. »Bien que les déterminants sociaux de la

\section{POINTS CLÉS}

- Le racisme, qui demeure répandu au Canada, est source d'inégalités sous-étudiées en santé.

- Les Instituts de recherche en santé du Canada, principal organisme de financement de la recherche en santé au pays, pourraient remédier à ce problème par la création d'un Institut du racisme et de ses effets sur la santé.

- La recherche en santé au Canada devrait commencer à envisager le racisme comme un déterminant de la santé et de reconnaître que l'amalgame des enjeux de racisme avec les préoccupations auxquelles se butent les populations immigrantes est désuet.

santé tels que le revenu et l'immigration ou le statut scolaire fassent souvent l'objet de recherches, l'influence de la race ou du racisme sur la santé n'ont pas fait l'objet d'études approfondies au Canada.

Nos travaux de recherche sur ce phénomène en santé au Canada, quoique relativement limités, sont éloquents ${ }^{5,6}$. Ainsi, lorsqu'il est ici question de race, nous reconnaissons qu'il s'agit d'une construction sociale se rapportant au phénomène au cours duquel certaines personnes deviennent racisées par l'effet conjugué au cours d'une vie de traitements, d'accès aux ressources et de possibilités différentielles systématiques ${ }^{7}$. Le concept de race est parfois confondu avec une conception génétique erronée qui attribue des dissemblances observées entre les races à de prétendues différences génétiques. La science a toutefois prouvé depuis longtemps que ce raisonnement était lacunaire ${ }^{8,9}$. Au Canada, des disparités raciales et ethniques ont été constatées pour plusieurs problèmes, notamment les maladies cardiovasculaires, le cancer et le diabète. La plupart des recherches actuelles se concentrent malgré tout sur les populations immigrantes, dont l'expérience distincte ne recoupe qu'à l'occasion celle des minorités visibles nées au pays. Si nous avons à cœur la lutte contre les inégalités raciales, nous devons augmenter substantiellement la quantité 
et la qualité des recherches menées sur ce sujet. L'absence de financement et d'infrastructures de soutien à la recherche sur les conséquences du racisme, y compris pour la collecte de donnée, constitue un obstacle fondamental à l'atteinte de cet objectif.

Notre proposition? Que le Canada s'engage dans la recherche consacrée aux effets du racisme et des initiatives antiracistes sur la santé en se dotant d'un Institut du racisme et de ses effets sur la santé au sein des IRSC. Son infrastructure de recherche pourrait être inspirée de celles de l'Institut de la santé des femmes et des hommes et de l'Institut de la santé des Autochtones, 2 bons exemples de l'importance de se doter d'instituts thématiques, adaptés à des populations en particulier. Les instituts des IRSC sont des réseaux de chercheurs qui se mobilisent autour d'enjeux précis pour orienter les futures recherches. Chapeautés et soutenus par les IRSC, ils élaborent des plans stratégiques et des objectifs connexes, bénéficient de l'expertise de comités consultatifs, mènent et appuient des initiatives d'application des connaissances et fournissent du financement pour la recherche, de la formation et des outils explicites aux chercheurs canadiens. L'Institut de la santé des Autochtones a fait participer la communauté à l'élaboration de priorités de recherche en santé impliquant les populations autochtones et a collaboré avec d'autres instituts des IRSC à la création d'initiatives de recherches ciblant les besoins des communautés autochtones ${ }^{10}$. Quant à l'Institut de la santé des femmes et des hommes, il a créé des initiatives de recherche interinstituts, a élaboré des modules de formation obligatoires et est devenu un chef de file de l'intégration du sexe et du genre pour améliorer la recherche en santé ${ }^{11}$.

Comme le racisme structurel est un phénomène mondial, nous pouvons aussi nous inspirer d'exemples à l'international. Aux États-Unis, les National Institutes of Health se sont dotés en 2010 d'un institut national de santé des minorités et des disparités en santé, institut qui a d'abord été un bureau (1990), puis un centre (2000). Depuis sa création, celui-ci a accordé plus de 1200 subventions de recherche. Il finance actuellement des recherches menées dans 200 sites à l'échelle nationale et internationale ${ }^{12}$.

Comme nous l'avons indiqué précédemment, il est trop tôt pour déduire que les enjeux concernant le racisme structurel et ses répercussions sur la santé sont moins prononcés au Canada qu'ailleurs, surtout compte tenu du manque de preuves tangibles du contraire.

Les IRSC ont récemment publié une lettre destinée aux scientifiques canadiens pour faire état des efforts actuels et à venir visant à s'attaquer à des enjeux de racisme structurel ${ }^{13}$. Le discours actuel est axé sur l'accroissement de la diversité des travailleurs en recherche et la mise en œuvre d'initiatives transinstituts. Ces mesures sont bienvenues, mais demeurent insuffisantes. La lutte contre le racisme structurel nécessite une planification et des mesures coordonnées et stratégiques, de même que le type de ressources intellectuelles et financières que posséderait un institut des IRSC dédié à cette problématique. Le Canada ne possède pas encore le champ et le niveau d'expertise nécessaire pour s'attaquer véritablement à des problèmes touchant le racisme et ses effets sur la santé dans les différents instituts. Toutefois, cet objectif est atteignable : le financement d'un institut permettrait d'offrir des formations poussées à celles et ceux qui souhaitent poursuivre une carrière dans ce domaine de recherche, de même que des formations obligatoires pour tous les chercheurs, comme celles sur le sexe et le genre, pour mieux tenir compte, de manière générale, du racisme et de ses effets sur la santé dans tous les domaines de recherche.

Chercheurs, praticiens et décideurs de la santé ne devraient pas reculer devant les véritables enjeux de l'étude des répercussions du racisme au Canada. Il est maintenant temps d'envisager le racisme comme un déterminant de la santé et de reconnaître que l'amalgame des enjeux de racisme avec les préoccupations auxquelles se butent les populations immigrantes est désuet. Il faut nommer le problème du racisme pour pouvoir créer des solutions visant à le régler directement. Si les structures du passé ont mené à des systèmes biaisés, le Canada doit en créer de nouvelles qui sont assez financées et appuyées pour commencer l'énorme travail de correction d'inégalités persistantes.

\section{Références}

1. Williams D. When it comes to systemic racism, history belies your words, Mr. Legault. CBC News Montreal 2020 June 13. Accessible ici : www.cbc.ca/ news/canada/montreal/systemic-racism-quebec-historian-1.5608397 (consulté le 10 nov. 2020).

2. Cooper A. The hanging of Angélique: the untold story of Canadian slavery and the burning of Old Montréal. Athens (GA): University of Georgia Press; 2007.

3. Human Rights Council; Working Group of Experts on People of African Descent. Report of the Working Group of Experts on People of African Descent on its mission to Canada. New York: United Nations Dag Hammarskjöld Library; 2017.

4. Advocacy position statements: racism and public health. Ottawa: Canadian Public Health Association; 2018.

5. Khan MM, Kobayashi K, Vang ZM, et al. Are visible minorities invisible in Canadian health data and research? A scoping review. Int J Migr Health Soc Care 2017;13:126-43.

6. Nestel S. Color coded health care: the impact of race and racism on Canadians health. Toronto: Wellesley Institute; 2012.

7. Sharma M, Kuper A. The elephant in the room: talking race in medical education. Adv Health Sci Educ Theory Pract 2017;22:761-4.

8. Boyd RW, Lindo EG, Weeks LD, et al. On racism: a new standard for publishing on racial health inequities [Blog]. Health Aff (Millwood) 2020 July 2. Accessible ici : www.healthaffairs.org/do/10.1377/hblog20200630.939347/full/ (consulté le 10 nov. 2020).

9. Mersha TB, Beck AF. The social, economic, political, and genetic value of race and ethnicity in 2020. Hum Genomics 2020;14:37.

10. Canadian Institutes of Health Research. Institute of Indigenous Peoples' Health Strategic Plan 2019-2024. Ottawa: Canadian Institutes of Health Research; 2019.

11. Canadian Institutes of Health Research Institute of Gender and Health. Science is better with sex and gender: Strategic Plan 2018-2023. Ottawa: Canadian Institutes of Health Research; 2018.

12. National Institute on Minority Health and Health Disparities Fact Sheet Bethesda (MD): National Institutes of Health; 2020.

13. Clifford T. CIHR's commitment to enhancing equity, diversity, and inclusion in the research funding system. Ottawa: Canadian Institutes of Health Research; modified 2020 Oct. 14. Accessible ici : cihr-irsc.gc.ca/e/52174.html (consulté le 15 oct. 2020). 
Intérêts concurrents : Arjumand Siddiqi est titulaire de la Chaire de recherche du Canada sur l'équité en matière de santé des populations, et Aisha Lofters est titulaire d'une chaire en science de la mise en œuvre au Centre Peter Gilgan pour les cancers féminins de l'Hôpital Women's College en partenariat avec la Société canadienne du cancer. Aucun autre intérêt concurrent n'a été déclaré.

Cet article a été révisé par des pairs.

Affiliations : Département de médecine sociale et préventive (Datta), Université de Montréal; Carrefour de l'innovation et de l'évaluation en santé (Datta), CRCHUM, Montréal, Qc; Service de médecine (Datta), Centre médical CedarsSinai, Los Angeles, É.-U.; École de santé publique Dalla Lana (Siddiqi), Université de
Toronto; Institut de recherche de l'Hôpital pour enfants malades (SickKids) (Siddiqi); Hôpital Women's College (Lofters); Département de médecine familiale et communautaire (Lofters), Faculté de médecine, Université de Toronto, Toronto, Ont.

Collaborateurs : Toutes les auteures ont contribué à la conception du travail, ont rédigé le manuscrit, en ont révisé de façon critique le contenu intellectuel important; elles ont donné leur approbation finale pour la version destinée à être publiée et assument l'entière responsabilité de tous les aspects du travail.

Propriété intellectuelle du contenu : Il s'agit d'un article en libre accès distribué conformément aux modalités de la licence Creative Com- mons Attribution (CC BY-NC-ND 4.0), qui permet l'utilisation, la diffusion et la reproduction dans tout médium à la condition que la publication originale soit adéquatement citée, que l'utilisation se fasse à des fins non commerciales (c.-à-d., recherche ou éducation) et qu'aucune modification ni adaptation n'y soit apportée. Voir : https://creativecommons.org/ licenses/by-nc-nd/4.0/deed.fr.

Remerciements : Les auteures remercient la $\mathrm{D}^{\text {re }}$ Onye Nnorom, présidente de la Black Physicians' Association of Ontario, d'avoir donné son avis sur une version précédente de ce commentaire.

Correspondance : Geetanjali Datta, geetanjali.datta@cshs.org 